\title{
NONCONTACT SUSPENDING AND TRANSPORTING PLANAR OBJECTS BY USING ACOUSTIC LEVITATION
}

\author{
Yoshiki Hashimoto (Kaijo Corporation) Yoshikazu Koike \\ and Sadayuki Ueha, Non-members \\ (Precision and Intelligence Laboratory, Tokyo institute of Technology)
}

keywords : acoustic levitation, ultrasonic, vibration plate, transportation, non-contact.

\section{INTRODUCTION}

A near field acoustic levitation phenomenon was recently reported by the authors where a several kilogram specimen can be stably levitated several tens microns apart from a rectangular flexural vibration plate ${ }^{1) 2}$. Any specimens can be levitated regardless of their material if the bottom surfaces are flat.

In this report, a contactless transportation system consisting of a suspending device and a transportation actuator is proposed. The near field acoustic levitation is used for the former while a conventional pulse motor actuator is used for the later part.

A trial-made non-contact transportation system is proved to transport a 8-inch silicon wafer successfully.

\section{CONFIGURATION OF THE PROPOSED SYSTEM}

Figure 1 shows the basic configuration of the proposed non-contact transportation system. The system consists of two parts, that is, a suspending and a transporting parts. The former, whose role is to levitate an object stably, consists of a circular vibrating plate, a horn, a transducer and an electric power source. A $3 \mathrm{~mm}$ thick circular vibrating plate $206 \mathrm{~mm}$ in diameter is connected to the horn with a countersunk screw so that the radiation surface of the plate is made flat. The plate is vibrated in flexural mode at its resonance. The horn is connected to a $20 \mathrm{kHz}$ bolt-clamped Langevin transducer. The vibration system is mounted on the linear actuator and is transported together with a levitated specimen on it. As the linear actuator a conventional pulse motor actuator (IA-03WX-60-500, THK, Japan) is used whose transportation distance and the maximum speed are $50 \mathrm{~cm}$ and $10 \mathrm{~cm} / \mathrm{s}$, respectively.

\section{LEVITATION FORCE BY THE VIBRATING PLATE.}

The vibration distribution of the circular duralumin plate driven at a frequency of $19.28 \mathrm{kHz}$ was measured by a high-frequency laser displacement meter and plotted in Fig.2. A concentric flexural vibration mode was observed. At the center of the vibrating plate, which corresponds to the countersunk screw, the cross recess is observed on the head. This is due to exceeding the measurement range

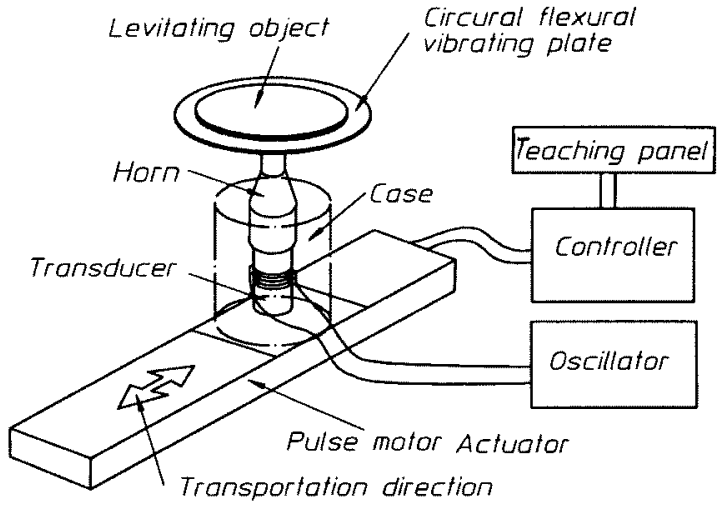

Fig.1 Basic configuration of non-contact transportation system.

of the laser displacement meter. Therefore, the measurement was impossible at the center and zero displacement was indicated. However, the vibration distribution obviously shows a typical Besselian.

Since an antinode is generated at the rim of the vibrating plate, the radiation pressure induces centripetal viscous force which works against sliding a 8-inch silicon wafer off the edge of the plate. This characteristic is convenient for the retainability, or holding an object during transportation.

According with the non-linear acoustic theory ${ }^{3)}$, the levitation force is expressed by the equation (1)

$$
F=\pi R^{2}\left(J_{0}^{2}(k R)+J_{1}^{2}(k R)\right) \frac{1+\gamma}{4} \rho_{a} c_{a}^{2} \frac{a_{o}^{2}}{h^{2}}
$$

where $R$ is the diameter of the levitation object, $J_{0}(k R)$ the Bessel-function of the first kind of order zero, $J_{l}(k R)$ the Bessel- function of the first kind of first, $\gamma$ the ratio of specific heats of the gas, $\mathrm{k}$ the wave number, $\rho_{a}$ the density of the gas, $c_{a}$ the sound speed of the gas, $a_{0}$ the amplitude displacement of the vibrating plate, and the $h$ levitation 
distance respectively.

If the equation is normalized by the bottom surface area of the levitation object $\pi R^{2}$, this value is equal to the weight per unit area of the levitating object.

\section{EXPERIMENTAL RESULTS}

\subsection{LEVITATION DISTANCE VERSUS DISPLACEMENT AMPLITUDE}

Using the equipment shown in Fig.1 and a 8-inch silicon wafer with a weight of $52.9 \mathrm{~g}$ (weight per unit area $1.7 \mathrm{~kg} / \mathrm{m}^{2}$ ), a levitation experiment was carried out. Figure 3 shows the levitation distance as a function of displacement amplitude.

According to the results, the levitation distance was proportional to the displacement amplitude, and the calculation results approximately agree with the experimental results. The levitation distance of $249 \mu \mathrm{m}$, for example, was obtained for a displacement amplitude of $30 \mu \mathrm{m}$.

\subsection{NON-CONTACT TRANSPORTARION}

The radial positioning fluctuation of a levitated Silicone wafer was measured with a laser displacement meter (amplifier LC-2400, sensor LC-2450), where the vibration amplitude at the center of the vibrating plate is $30 \mu \mathrm{m}_{\mathrm{pp}}$. As a result of measurement, the fluctuation of levitating 8-inch silicon wafer is within $\pm 2.5 \mathrm{~mm}$.

Using the non-contact transportation equipment shown in Figure 1, a levitating 8-inch silicon wafer together with the vibrating system was tried to be transported by the pulse motor actuator.

As a result, a transportation speed of up to $10 \mathrm{~cm} / \mathrm{s}$ was marked. The criteria is given by the actuator used for the experiment and can be improved if a superior linear actuator is used.

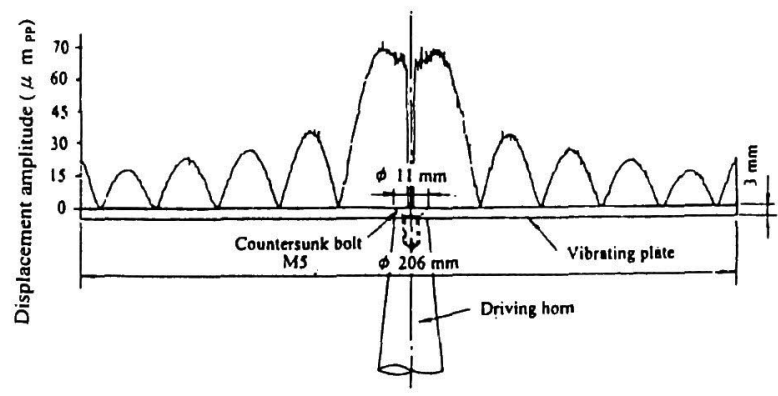

Fig.2 Vibration distribution of the circular vibration plate

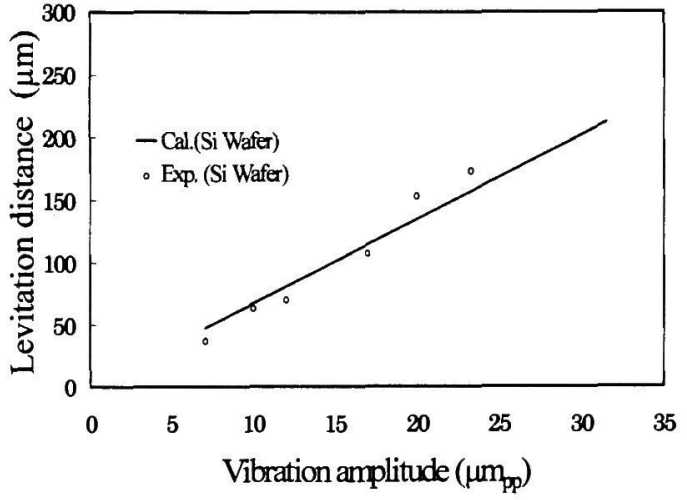

Fig.3 Displacement amplitude versus levitation distance

\section{CONCLUSION}

A non-contact transportation system using the near-field acoustic levitation was proposed and proved to work well. A 8-inch silicon wafer is successfully transported by the trial-made apparatus.

The optimum relation between the size of levitated specimen and the diameter of vibrating plate is under study.

\section{REFERENCES}

(1) Y.Hashimoto, Y.Koike and S.Ueha, 1st W.C.U., pp.835-836 (1995.9)

(2) Y.Hashimoto, Y.Koike and S.Ueha, J.Acoust.Soc.Am., 100(4) 2058-2061(1996.10)

(3) Boa-The Chu and Robert E. Apfel J.Acoust.Soc.Am., 72(6) 1673-1687(1982.12)

Yoshiki Hashimoto (non-member) was born on January 14,

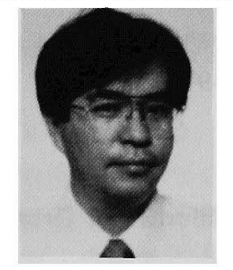
1959. He received the $B$. Eng. degree in electric engineering from Ashikaga Institute of Technology in 1981. Since 1981, he has been with Kaijo Co., Ltd., where he has been engaged in the development and application on high power ultrasonic systems.

Yoshikazu Koike

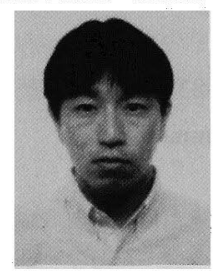

Sdayuki Ueha

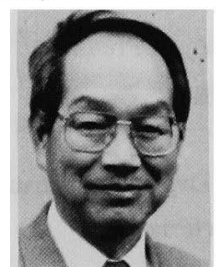

(non-member) was born on February 28, 1943. He received the D. Eng. degree in e lectric engineering from Tokyo Institute of Technology in 1970. Now, he is a full p rofessor of Tokyo Institute of Technology. A nd Dr. Ueha is currently President of the A coustic Society of Japan since May,1997. 